\title{
ON MULTIVALUED NONLINEAR VARIATIONAL INCLUSION PROBLEMS WITH $(A, \eta)$-ACCRETIVE MAPPINGS IN BANACH SPACES
}

\author{
HENG-YOU LAN
}

Received 20 January 2006; Revised 12 May 2006; Accepted 15 May 2006

Based on the notion of $(A, \eta)$-accretive mappings and the resolvent operators associated with $(A, \eta)$-accretive mappings due to Lan et al., we study a new class of multivalued nonlinear variational inclusion problems with $(A, \eta)$-accretive mappings in Banach spaces and construct some new iterative algorithms to approximate the solutions of the nonlinear variational inclusion problems involving $(A, \eta)$-accretive mappings. We also prove the existence of solutions and the convergence of the sequences generated by the algorithms in $q$-uniformly smooth Banach spaces.

Copyright (c) 2006 Heng-You Lan. This is an open access article distributed under the Creative Commons Attribution License, which permits unrestricted use, distribution, and reproduction in any medium, provided the original work is properly cited.

\section{Introduction}

Recently, in order to study extensively variational inequalities and variational inclusions, which are providing mathematical models to some problems arising in economics, mechanics, and engineering science, Ding [1], Huang and Fang [10], Fang and Huang [3], Verma [14, 15], Fang and Huang [4, 5], Huang and Fang [9], Fang et al. [2] have introduced the concepts of $\eta$-subdifferential operators, maximal $\eta$-monotone operators, generalized monotone operators (named $H$-monotone operators), $A$-monotone operators, $(H, \eta)$-monotone operators in Hilbert spaces, $H$-accretive operators, generalized $m$ accretive mappings and $(H, \eta)$-accretive operators in Banach spaces, and their resolvent operators, respectively. Very recently, Fang et al. [7], studied the $(H, \eta)$-monotone operators in Hilbert spaces, which are a special case of $(H, \eta)$-accretive operator [2]. Some works are motivated by this work and some related works. The iterative algorithms for the variational inclusions with $H$-accretive operators can be found in the paper [6]. Further, Lan et al. [11] introduced a new concept of $(A, \eta)$-accretive mappings, which generalizes the existing monotone or accretive operators, studied some properties of $(A, \eta)$-accretive mappings, and defined resolvent operators associated with $(A, \eta)$-accretive mappings. Moreover, by using the resolvent operator technique, many authors constructed some

Hindawi Publishing Corporation Journal of Inequalities and Applications Volume 2006, Article ID 59836, Pages 1-12 DOI 10.1155/JIA/2006/59836 
perturbed iterative algorithms for some nonlinear variational inclusions in Hilbert space or Banach spaces. For more detail, see, for example, $[1-8,10,11,14,15]$ and the references therein.

On the other hand, Lan et al. [12] introduced and studied some new iterative algorithms for solving a class of nonlinear variational inequalities with multivalued mappings in Hilbert spaces, and gave some convergence analysis of iterative sequences generated by the algorithms.

Motivated and inspired by the above works, the purpose of this paper is to introduce the notion of $(A, \eta)$-accretive mappings and the resolvent operators associated with $(A, \eta)$-accretive mappings due to Lan et al., to study a new class of multivalued nonlinear variational inclusion problems with $(A, \eta)$-accretive mappings in Banach spaces, and to construct some new iterative algorithms to approximate the solutions of the nonlinear variational inclusion problems involving $(A, \eta)$-accretive mappings. We also prove the existence of solutions and the convergence of the sequences generated by the algorithms in $q$-uniformly smooth Banach spaces.

\section{Preliminaries}

Let $X$ be a real Banach space with dual space $X^{*}$, let $\langle\cdot, \cdot\rangle$ be the dual pair between $X$ and $X^{*}$, let $2^{X}$ denote the family of all the nonempty subsets of $X$, and let $\mathrm{CB}(X)$ denote the family of all nonempty closed bounded subsets of $X$. The generalized duality mapping $J_{q}: X \rightarrow 2^{X^{*}}$ is defined by

$$
J_{q}(x)=\left\{f^{*} \in X^{*}:\left\langle x, f^{*}\right\rangle=\|x\|^{q},\left\|f^{*}\right\|=\|x\|^{q-1}\right\}, \quad \forall x \in X
$$

where $q>1$ is a constant. In particular, $J_{2}$ is the usual normalized duality mapping. It is known that, in general, $J_{q}(x)=\|x\|^{q-2} J_{2}(x)$ for all $x \neq 0$, and $J_{q}$ is single valued if $X^{*}$ is strictly convex, and if $X=\mathscr{H}$, the Hilbert space, then $J_{2}$ becomes the identity mapping on H.

The modulus of smoothness of $X$ is the function $\rho_{X}:[0, \infty) \rightarrow[0, \infty)$ defined by

$$
\rho_{X}(t)=\sup \left\{\frac{1}{2}(\|x+y\|+\|x-y\|)-1:\|x\| \leq 1,\|y\| \leq t\right\}
$$

A Banach space $X$ is called uniformly smooth if

$$
\lim _{t \rightarrow 0} \frac{\rho_{X}(t)}{t}=0
$$

$X$ is called $q$-uniformly smooth if there exists a constant $c>0$ such that

$$
\rho_{X}(t) \leq c t^{q}, \quad q>1
$$

Note that $J_{q}$ is single valued if $X$ is uniformly smooth, and Hilbert space and $L_{p}$ (or $l_{p}$ ) $(2 \leq p<\infty)$ spaces are 2 -uniformly Banach spaces. In what follows, we will denote the single valued generalized duality mapping by $J_{q}$. 
In the study of characteristic inequalities in $q$-uniformly smooth Banach spaces, $\mathrm{Xu}$ [16] proved the following result.

Lemma 2.1. Let $X$ be a real uniformly smooth Banach space. Then $X$ is $q$-uniformly smooth if and only if there exists a constant $c_{q}>0$ such that for all $x, y \in X$,

$$
\|x+y\|^{q} \leq\|x\|^{q}+q\left\langle y, J_{q}(x)\right\rangle+c_{q}\|y\|^{q} .
$$

Definition 2.2. Let $X$ be a real $q$-uniformly smooth Banach space and let $T, A: X \rightarrow X$ be two single-valued mappings. $T$ is said to be

(i) accretive if

$$
\left\langle T(x)-T(y), J_{q}(x-y)\right\rangle \geq 0, \quad \forall x, y \in X
$$

(ii) strictly accretive if $T$ is accretive and $\left\langle T(x)-T(y), J_{q}(x-y)\right\rangle=0$ if and only if $x=y$;

(iii) $r$-strongly accretive if there exists a constant $r>0$ such that

$$
\left\langle T(x)-T(y), J_{q}(x-y)\right\rangle \geq r\|x-y\|^{q}, \quad \forall x, y \in X
$$

(iv) $\gamma$-strongly accretive with respect to $A$ if there exists a constant $\gamma>0$ such that

$$
\left\langle T(x)-T(y), J_{q}(A(x)-A(y))\right\rangle \geq \gamma\|x-y\|^{q}, \quad \forall x, y \in X
$$

(v) $m$-relaxed cocoercive with respect to $A$ if there exists a constant $m>0$ such that

$$
\left\langle T(x)-T(y), J_{q}(A(x)-A(y))\right\rangle \geq-m\|T(x)-T(y)\|^{q}, \quad \forall x, y \in X ;
$$

(vi) $(\alpha, \xi)$-relaxed cocoercive with respect to $A$ if there exist constants $\alpha, \xi>0$ such that

$$
\left\langle T(x)-T(y), J_{q}(A(x)-A(y))\right\rangle \geq-\alpha\|T(x)-T(y)\|^{q}+\xi\|x-y\|^{q}, \quad \forall x, y \in X
$$

(vii) $s$-Lipschitz continuous if there exists a constant $s>0$ such that

$$
\|T(x)-T(y)\| \leq s\|x-y\|, \quad \forall x, y \in X
$$

Remark 2.3. When $X=\mathscr{H}$, (i)-(iv) of Definition 2.2 reduce to the definitions of monotonicity, strict monotonicity, strong monotonicity, and strong monotonicity with respect to $A$, respectively (see $[1,3,5]$ ). 
4 On multivalued nonlinear variational inclusion problems

Example 2.4. Consider a nonexpansive mapping $T: \mathscr{H} \rightarrow \mathscr{H}$. If we set $F=I-T$, where $I$ is the identity mapping, then $F$ is $(1 / 2)$-cocoercive.

Proof. For any two elements $x, y \in \mathscr{H}$, we have

$$
\begin{aligned}
\|F(x)-F(y)\|^{2} & =\|(I-T)(x)-(I-T)(y)\|^{2} \\
& =\langle(I-T)(x)-(I-T)(y),(I-T)(x)-(I-T)(y)\rangle \\
& \leq 2\left[\|x-y\|^{2}-\langle x-y, T(x)-T(y)\rangle\right] \\
& =2\langle x-y, F(x)-F(y)\rangle,
\end{aligned}
$$

that is, $F$ is $(1 / 2)$-cocoercive.

Example 2.5. Consider a projection $P: \mathscr{H} \rightarrow C$, where $C$ is a nonempty closed convex subset of $\mathcal{H}$. Then $P$ is 1 -cocoercive since $P$ is nonexpansive.

Proof. For any $x, y \in \mathscr{H}$, we have

$$
\begin{aligned}
\|P(x)-P(y)\|^{2} & =\langle P(x)-P(y), P(x)-P(y)\rangle \\
& \leq\langle x-y, P(x)-P(y)\rangle .
\end{aligned}
$$

Thus, $P$ is 1 -cocoercive.

Example 2.6. An $r$-strongly monotone (and hence $r$-expanding) mapping $T: \mathscr{H} \rightarrow \mathscr{H}$ is $\left(r+r^{2}, 1\right)$-relaxed cocoercive with respect to $I$.

Proof. For any two elements $x, y \in \mathscr{H}$, we have

$$
\begin{gathered}
\|T(x)-T(y)\| \geq r\|x-y\|, \\
\langle T(x)-T(y), x-y\rangle \geq r\|x-y\|^{2},
\end{gathered}
$$

and so

$$
\|T(x)-T(y)\|^{2}+\langle T(x)-T(y), x-y\rangle \geq\left(r+r^{2}\right)\|x-y\|^{2},
$$

that is, for all $x, y \in \mathscr{H}$, we get

$$
\langle T(x)-T(y), x-y\rangle \geq(-1)\|T(x)-T(y)\|^{2}+\left(r+r^{2}\right)\|x-y\|^{2} .
$$

Therefore, $T$ is $\left(r+r^{2}, 1\right)$-relaxed cocoercive with respect to $I$.

Remark 2.7. Clearly, every $m$-cocoercive mapping is $m$-relaxed cocoercive, while each $r$-strongly monotone mapping is $\left(r+r^{2}, 1\right)$-relaxed cocoercive with respect to $I$. 
Definition 2.8. A single valued mapping $\eta: X \times X \rightarrow X$ is said to be $\tau$-Lipschitz continuous if there exists a constant $\tau>0$ such that $\|\eta(x, y)\| \leq \tau\|x-y\|$, for all $x, y \in X$.

Definition 2.9. Let $X$ be a real $q$-uniformly smooth Banach space and let $\eta: X \times X \rightarrow X$ and $A, H: X \rightarrow X$ be single valued mappings. A set-valued mapping $M: X \rightarrow 2^{X}$ is said to be

(i) accretive if

$$
\left\langle u-v, J_{q}(x-y)\right\rangle \geq 0, \quad \forall x, y \in X, u \in M(x), v \in M(y)
$$

(ii) $\eta$-accretive if

$$
\left\langle u-v, J_{q}(\eta(x, y))\right\rangle \geq 0, \quad \forall x, y \in X, u \in M(x), v \in M(y)
$$

(iii) strictly $\eta$-accretive if $M$ is $\eta$-accretive and equality holds if and only if $x=y$;

(iv) $r$-strongly $\eta$-accretive if there exists a constant $r>0$ such that

$$
\left\langle u-v, J_{q}(\eta(x, y))\right\rangle \geq r\|x-y\|^{q}, \quad \forall x, y \in X, u \in M(x), v \in M(y)
$$

(v) $\alpha$-relaxed $\eta$-accretive if there exists a constant $\alpha>0$ such that

$$
\left\langle u-v, J_{q}(\eta(x, y))\right\rangle \geq-\alpha\|x-y\|^{q}, \quad \forall x, y \in X, u \in M(x), v \in M(y)
$$

(vi) $m$-accretive if $M$ is accretive and $(I+\rho M)(X)=X$ for all $\rho>0$, where $I$ denotes the identity operator on $X$;

(vii) generalized $m$-accretive if $M$ is $\eta$-accretive and $(I+\rho M)(X)=X$ for all $\rho>0$;

(viii) $H$-accretive if $M$ is accretive and $(H+\rho M)(X)=X$ for all $\rho>0$;

(ix) $(H, \eta)$-accretive if $M$ is $\eta$-accretive and $(H+\rho M)(X)=X$ for every $\rho>0$.

Remark 2.10. (1) The class of generalized $m$-accretive operators was first introduced by Huang and Fang [9], and includes that of $m$-accretive operators as a special case. The class of $H$-accretive operators was first introduced and studied by Fang and Huang [5], and also includes that of $m$-accretive operators as a special case.

(2) When $X=\mathscr{H}$, (i)-(ix) of Definition 2.9 reduce to the definitions of monotone operators, $\eta$-monotone operators, strictly $\eta$-monotone operators, strongly $\eta$-monotone operators, relaxed $\eta$-monotone operators, maximal monotone operators, maximal $\eta$ monotone operators, $H$-monotone operators, and $(H, \eta)$-monotone operators, respectively.

Definition 2.11. Let $A: X \rightarrow X$ and $\eta: X \times X \rightarrow X$ be two single-valued mappings. A multivalued mapping $M: X \rightarrow 2^{X}$ is called $(A, \eta)$-accretive if $M$ is $m$-relaxed $\eta$-accretive and $(A+\rho M)(X)=X$ for every $\rho>0$. 
Remark 2.12. For appropriate and suitable choices of $m, A, \eta$, and $X$, it is easy to see that Definition 2.11 includes a number of definitions of monotone operators and accretive operators (see [11]).

Proposition 2.13 [11]. Let $A: X \rightarrow X$ be a $r$-strongly $\eta$-accretive mapping, let $M: X \rightarrow 2^{X}$ be an $(A, \eta)$-accretive mapping. Then the operator $(A+\rho M)^{-1}$ is single valued.

Remark 2.14. Proposition 2.13 generalizes and improves [3, Theorem 2.1], [5, Theorem 2.2], [4, Theorem 3.2], [2, Theorem 3.2], [10, (2) of Theorem 2.1], and [9], respectively,

Based on Proposition 2.13, we can define the resolvent operator $R_{\eta, M}^{\rho, A}$ associated with an $(A, \eta)$-accretive mapping $M$ as follows.

Definition 2.15. Let $A: X \rightarrow X$ be a strictly $\eta$-accretive mapping and let $M: X \rightarrow 2^{X}$ be an $(A, \eta)$-accretive mapping. The resolvent operator $R_{\eta, M}^{\rho, A}: X \rightarrow X$ is defined by

$$
R_{\eta, M}^{\rho, A}(x)=(A+\rho M)^{-1}(x), \quad \forall x \in X .
$$

Remark 2.16. Resolvent operators associated with $(A, \eta)$-accretive mappings include as special cases the corresponding resolvent operators associated with $(H, \eta)$-accretive mappings [2], $(H, \eta)$-monotone operators $[4,7], H$-accretive operators $[5,6]$, generalized $m$-accretive operators [9], maximal $\eta$-monotone operators [10], $H$-monotone operators [3], $A$-monotone operators [14], $\eta$-subdifferential operators [1], the classical $m$-accretive, and maximal monotone operators [17].

Proposition 2.17 [11]. Let $X$ be a real q-uniformly smooth Banach space and let $\eta: X \times$ $X \rightarrow X$ be $\tau$-Lipschitz continuous, let $A: X \rightarrow X$ be an $r$-strongly $\eta$-accretive mapping, and let $M: X \rightarrow 2^{X}$ be an $(A, \eta)$-accretive mapping. Then the resolvent operator $R_{\eta, M}^{\rho, A}: X \rightarrow X$ is $\tau^{q-1} /(r-\rho m)$-Lipschitz continuous, that is,

$$
\left\|R_{\eta, M}^{\rho, A}(x)-R_{\eta, M}^{\rho, A}(y)\right\| \leq \frac{\tau^{q-1}}{r-\rho m}\|x-y\|, \quad \forall x, y \in X,
$$

where $\rho \in(0, r / m)$ is a constant.

Remark 2.18. Proposition 2.17 extends [2, Theorem 3.3] and [15, Lemma 2], and so extends [10, Theorem 2.2], [3, Theorem 2.2], [5, Theorem 2.3], [4, Theorem 3.3], [1, Theorem 2.2], and [9, Theorem 2.3].

Definition 2.19. Let $T: X \rightarrow 2^{X}$ be a set-valued mapping. For all $x, y \in X, T$ is said to be $\zeta$ - $\hat{\mathbf{H}}$-Lipschitz continuous, if there exists a constant $\zeta>0$ such that

$$
\hat{\mathbf{H}}(T(x), T(y)) \leq \zeta\|x-y\|, \quad \forall x, y \in X
$$

where $\hat{\mathbf{H}}: 2^{X} \times 2^{X} \rightarrow(-\infty,+\infty) \cup\{+\infty\}$ is the Hausdorff pseudometric, that is,

$$
\hat{\mathbf{H}}(D, B)=\max \left\{\sup _{x \in D} \inf _{y \in B}\|x-y\|, \sup _{x \in B} \inf _{y \in D}\|x-y\|\right\}, \quad \forall D, B \in 2^{X} .
$$


Note that if the domain of $\hat{\mathbf{H}}$ is restricted to closed bounded subsets $\mathrm{CB}(X)$, then $\hat{\mathbf{H}}$ is the Hausdorff metric.

Let $f, g: X \rightarrow X$ and let $T: X \rightarrow 2^{X}$ be nonlinear mappings and let $M: X \rightarrow 2^{X}$ be an $(A, \eta)$-accretive mapping with $g(X) \cap \operatorname{Dom} M \neq \varnothing$. For any given $\lambda>0$, the following multivalued nonlinear variational inclusion problem will be considered.

Find $x \in X$ such that $u \in T(x)$ and

$$
0 \in f(x)+u+\lambda M(g(x))
$$

Example 2.20. (1) If $g=I$ and $\lambda=1$, then a special case of the problem (2.25) is determining elements $x \in X$ and $u \in T(x)$ such that

$$
0 \in f(x)+u+M(x)
$$

(2) Further, if $X=X^{*}=\mathscr{H}, \eta(x, y)=x-y$, and $M=\Delta \varphi$, where $\Delta \varphi$ denotes the subdifferential of a proper convex lower semicontinuous function $\varphi$ on $\mathscr{H}$, then the problem (2.26) becomes the following classical variational inequality.

Find $x \in X$ such that

$$
\langle f(x)+u, y-x\rangle+\varphi(y)-\varphi(x) \geq 0, \quad \forall y \in X
$$

(3) If $M(x)=\partial \delta_{K}(x)$ for all $x \in K$, where $K$ is a nonempty closed convex subset of $X$, and $\partial \delta_{K}$ denotes indicator function of $K$, then the problem (2.27) becomes to determining elements $x \in K$ and $u \in T(x)$ such that

$$
\langle f(x)+u, y-x\rangle \geq 0, \quad \forall y \in X
$$

which is the problem studied by Lan et al. [12].

Remark 2.21. For appropriate and suitable choices of $f, T, M, g$, and $X$, it is easy to see that the problem (2.25) includes a number of quasi-variational inclusions, generalized quasi-variational inclusions, quasi-variational inequalities, implicit quasi-variational inequalities studied by many authors as special cases, see, for example, $[1,5,8,12,17]$ and the references therein.

\section{Iterative algorithms and convergence}

In this section, we firstly suggest and analyze a new iterative method for solving the multivalued nonlinear variational inclusion problem (2.25).

Lemma 3.1. Let $A: X \rightarrow X$ be r-strongly $\eta$-accretive, let $M: X \rightarrow 2^{X}$ be $(A, \eta)$-accretive, and let $T: X \rightarrow \mathrm{CB}(X)$ and $f: X \rightarrow X$ be any nonlinear mappings. If

$$
Q(x)=g(x)-R_{\eta, M}^{\rho \lambda, A}[A(g(x))-\rho(f+T)(x)],
$$


8 On multivalued nonlinear variational inclusion problems

where $R_{\eta, M}^{\rho \lambda, A}=(A+\rho \lambda M)^{-1}$ and $\rho>0$ is a constant, then the nonlinear variational inclusion problem (2.25) has a solution if and only if $0 \in Q(x)$.

Proof. It is obvious that "only if" part holds.

Now, if $0 \in Q(x)$, then there exists a $u \in T(x)$ such that

$$
g(x)=R_{\eta, M}^{\rho \lambda, A}[A(g(x))-\rho(f(x)+u)]
$$

From the definition of the resolvent operators associated with $(A, \eta)$-accretive mappings, we know that for any $u \in T(x)$,

$$
A(g(x))-\rho(f(x)+u) \in A(g(x))+\rho \lambda M(g(x))
$$

that is,

$$
0 \in f(x)+u+\lambda M(g(x))
$$

Therefore, $(x, u)$ is a solution of the problem (2.25). This completes the proof.

From Lemma 3.1, we can suggest the following iterative algorithm.

Algorithm 3.2. Let $\mu \in(0,1]$ be a constant, let $T: X \rightarrow 2^{X}$ be a multivalued mapping, and let $f: X \rightarrow X$ be a single-valued mapping. For given $x_{0} \in X, u_{0} \in T\left(x_{0}\right)$, let

$$
x_{1}=(1-\mu) x_{0}-\mu\left\{x_{0}-g\left(x_{0}\right)+R_{\eta, M}^{\rho \lambda, A}\left[A\left(g\left(x_{0}\right)\right)-\rho\left(f\left(x_{0}\right)+u_{0}\right)\right]\right\} .
$$

By Nadler's theorem [13], there exists $u_{1} \in T\left(x_{1}\right)$ such that

$$
\left\|u_{0}-u_{1}\right\| \leq(1+1) \hat{\mathbf{H}}\left(T\left(x_{0}\right), T\left(x_{1}\right)\right) .
$$

Set

$$
x_{2}=(1-\mu) x_{1}-\mu\left\{x_{1}-g\left(x_{1}\right)+R_{\eta, M}^{\rho \lambda, A}\left[A\left(g\left(x_{1}\right)\right)-\rho\left(f\left(x_{1}\right)+u_{1}\right)\right]\right\} .
$$

By induction, we can define sequences $\left\{x_{n}\right\}$ and $\left\{u_{n}\right\}$ inductively satisfying

$$
\begin{gathered}
x_{n+1}=(1-\mu) x_{n}-\mu\left\{x_{n}-g\left(x_{n}\right)+R_{\eta, M}^{\rho \lambda, A}\left[A\left(g\left(x_{n}\right)\right)-\rho\left(f\left(x_{n}\right)+u_{n}\right)\right]\right\}, \\
u_{n} \in T\left(x_{n}\right), \quad\left\|u_{n}-u_{n+1}\right\| \leq\left[1+(n+1)^{-1}\right] \hat{\mathbf{H}}\left(T\left(x_{n}\right), T\left(x_{n+1}\right)\right) .
\end{gathered}
$$


Algorithm 3.3. If $g \equiv I$ and $\lambda=\mu=1$, then Algorithm 3.2 can be written as follows:

$$
\begin{gathered}
x_{n+1}=R_{\eta, M}^{\rho, A}\left[A\left(x_{n}\right)-\rho\left(f\left(x_{n}\right)+u_{n}\right)\right], \\
u_{n} \in T\left(x_{n}\right), \quad\left\|u_{n}-u_{n+1}\right\| \leq\left[1+(n+1)^{-1}\right] \hat{\mathbf{H}}\left(T\left(x_{n}\right), T\left(x_{n+1}\right)\right) .
\end{gathered}
$$

We now discuss the existence of a solution of the problem (2.25) and the convergence of Algorithm 3.2.

Theorem 3.4. Let $X$ be a q-uniformly smooth Banach space and let $A: X \rightarrow X$ be $r$ strongly $\eta$-accretive and $\epsilon$-Lipschitz continuous, respectively. Suppose that $T: X \rightarrow \mathrm{CB}(X)$ is $\gamma$ - $\hat{\mathbf{H}}$-Lipschitz continuous, $\eta: X \times X \rightarrow X$ is $\tau$-Lipschitz continuous, and $M: X \rightarrow 2^{X}$ is $(A, \eta)$-accretive. Let $g$ be $(d, \alpha)$-relaxed cocoercive and $\beta$-Lipschitz continuous, let $f$ be $(e, \delta)$ relaxed cocoercive with respect to $g_{1}$ and $\sigma$-Lipschitz continuous, where $g_{1}: X \rightarrow X$ is defined by $g_{1}(x)=A \circ g(x)=A(g(x))$ for all $x \in X$. If there exists a constant $\rho \in(0, r / \lambda m)$ such that

$$
\begin{gathered}
k=\sqrt[q]{1-q \alpha+\left(c_{q}+d q\right) \beta^{q}}<1-\frac{\rho \gamma \tau^{q-1}}{r-\rho \lambda m}, \\
\epsilon^{q} \beta^{q}-q \rho \delta+q \rho e \sigma^{q}+c_{q} \rho^{q} \sigma^{q}<\left[(1-k)(r-\rho \lambda m) \tau^{1-q}-\rho \gamma\right]^{q},
\end{gathered}
$$

where $c_{q}$ is the constant as in Lemma 2.1, then the iterative sequences $\left\{x_{n}\right\}$ and $\left\{u_{n}\right\}$ generated by Algorithm 3.2 converge strongly to $x^{*}$ and $u^{*}$, respectively, and $\left(x^{*}, u^{*}\right)$ is a solution of problem (2.25).

Proof. It follows from (3.8) and Proposition 2.17 that

$$
\begin{aligned}
\| x_{n+1}- & x_{n} \| \\
= & \|(1-\mu) x_{n}-\mu\left\{x_{n}-g\left(x_{n}\right)+R_{\eta, M}^{\rho \lambda, A}\left[A\left(g\left(x_{n}\right)\right)-\rho\left(f\left(x_{n}\right)+u_{n}\right)\right]\right\} \\
& \quad-(1-\mu) x_{n-1}+\mu\left\{x_{n-1}-g\left(x_{n-1}\right)+R_{\eta, M}^{\rho \lambda, A}\left[A\left(g\left(x_{n-1}\right)\right)-\rho\left(f\left(x_{n-1}\right)+u_{n-1}\right)\right]\right\} \| \\
\leq & (1-\mu)\left\|x_{n}-x_{n-1}\right\|+\mu\left\|x_{n}-x_{n-1}-\left[g\left(x_{n}\right)-g\left(x_{n-1}\right)\right]\right\| \\
& +\mu\left\|R_{\eta, M}^{\rho \lambda, A}\left[A\left(g\left(x_{n}\right)\right)-\rho\left(f\left(x_{n}\right)+u_{n}\right)\right]-R_{\eta, M}^{\rho \lambda, A}\left[A\left(g\left(x_{n-1}\right)\right)-\rho\left(f\left(x_{n-1}\right)+u_{n-1}\right)\right]\right\| \\
\leq & (1-\mu)\left\|x_{n}-x_{n-1}\right\|+\mu\left\|x_{n}-x_{n-1}-\left[g\left(x_{n}\right)-g\left(x_{n-1}\right)\right]\right\| \\
& +\mu \frac{\tau^{q-1}}{r-\rho \lambda m}\left\|A\left(g\left(x_{n}\right)\right)-\rho\left(f\left(x_{n}\right)+u_{n}\right)-\left[A\left(g\left(x_{n-1}\right)\right)-\rho\left(f\left(x_{n-1}\right)+u_{n-1}\right)\right]\right\| \\
\leq & (1-\mu)\left\|x_{n}-x_{n-1}\right\|+\mu\left\|x_{n}-x_{n-1}-\left[g\left(x_{n}\right)-g\left(x_{n-1}\right)\right]\right\| \\
& +\frac{\mu \tau^{q-1}}{r-\rho \lambda m}\left\|A\left(g\left(x_{n}\right)\right)-A\left(g\left(x_{n-1}\right)\right)-\rho\left(f\left(x_{n}\right)-f\left(x_{n-1}\right)\right)\right\|+\frac{\mu \rho \tau^{q-1}}{r-\rho \lambda m}\left\|u_{n}-u_{n-1}\right\| .
\end{aligned}
$$


By the assumptions and Lemma 2.1, we know that

$$
\begin{aligned}
\| x_{n}- & x_{n-1}-\left(g\left(x_{n}\right)-g\left(x_{n-1}\right)\right) \|^{q} \\
\leq & \left\|x_{n}-x_{n-1}\right\|^{q}-q\left\langle g\left(x_{n}\right)-g\left(x_{n-1}\right), J_{q}\left(x_{n}-x_{n-1}\right)\right\rangle \\
& +c_{q}\left\|g\left(x_{n}\right)-g\left(x_{n-1}\right)\right\|^{q} \\
\leq & \left(1-q \alpha+\left(c_{q}+d q\right) \beta^{q}\right)\left\|x_{n}-x_{n-1}\right\|^{q}, \\
\left\|A\left(g\left(x_{n}\right)\right)-A\left(g\left(x_{n-1}\right)\right)-\rho\left(f\left(x_{n}\right)-f\left(x_{n-1}\right)\right)\right\|^{q} & \\
\leq & \left\|A\left(g\left(x_{n}\right)\right)-A\left(g\left(x_{n-1}\right)\right)\right\|^{q}+c_{q} \rho^{q}\left\|f\left(x_{n}\right)-f\left(x_{n-1}\right)\right\|^{q} \\
& -q \rho\left\langle f\left(x_{n}\right)-f\left(x_{n-1}\right), J_{q}\left(A\left(g\left(x_{n}\right)\right)-A\left(g\left(x_{n-1}\right)\right)\right)\right\rangle \\
\leq & \left(\epsilon^{q} \beta^{q}+c_{q} \rho^{q} \sigma^{q}\right)\left\|x_{n}-x_{n-1}\right\|^{q} \\
& -q \rho\left(-e\left\|f\left(x_{n}\right)-f\left(x_{n-1}\right)\right\|^{q}+\delta\left\|x_{n}-x_{n-1}\right\|^{q}\right) \\
\leq & \left(\epsilon^{q} \beta^{q}-q \rho \delta+q \rho e \sigma^{q}+c_{q} \rho^{q} \sigma^{q}\right)\left\|x_{n}-x_{n-1}\right\|^{q}, \\
\| u_{n}- & u_{n-1}\left\|\leq\left(1+n^{-1}\right) \hat{\mathbf{H}}\left(T\left(x_{n}\right), T\left(x_{n-1}\right)\right) \leq \gamma\left(1+n^{-1}\right)\right\| x_{n}-x_{n-1} \| .
\end{aligned}
$$

Combining (3.11)-(3.14), we have

$$
\left\|x_{n+1}-x_{n}\right\| \leq\left(1-\mu+\mu \theta_{n}\right)\left\|x_{n}-x_{n-1}\right\|,
$$

where

$$
\theta_{n}=\sqrt[q]{1-q \alpha+\left(c_{q}+d q\right) \beta^{q}}+\frac{\tau^{q-1} \sqrt[q]{\epsilon^{q} \beta^{q}-q \rho \delta+q \rho e \sigma^{q}+c_{q} \rho^{q} \sigma^{q}}}{r-\rho \lambda m}+\frac{\rho \gamma \tau^{q-1}\left(1+n^{-1}\right)}{r-\rho \lambda m} .
$$

Let $\theta=\sqrt[q]{1-q \alpha+\left(c_{q}+d q\right) \beta^{q}}+\tau^{q-1} \sqrt[q]{\epsilon^{q} \beta^{q}-q \rho \delta+q \rho e \sigma^{q}+c_{q} \rho^{q} \sigma^{q}} /(r-\rho \lambda m)+\rho \gamma \tau^{q-1} /$ $(r-\rho \lambda m)$. Then we know that

$$
\theta_{n} \downarrow \theta \quad \text { as } n \longrightarrow \infty
$$

From the condition (3.10), we know that $0<\theta<1$, and hence there exist an $n_{0}>0$ and $\theta_{0} \in(\theta, 1)$ such that $\theta_{n} \leq \theta_{0}$ for all $n \geq n_{0}$. Therefore, by (3.15), we have

$$
\left\|x_{n+1}-x_{n}\right\| \leq \theta_{0}\left\|x_{n}-x_{n-1}\right\|, \quad n \geq n_{0}
$$

It follows from (3.18) that

$$
\left\|x_{n+1}-x_{n}\right\| \leq \theta_{0}^{n-n_{0}}\left\|x_{n_{0}+1}-x_{n_{0}}\right\|, \quad n \geq n_{0}
$$


Hence, for any $m \geq n>n_{0}$, it follows that

$$
\left\|x_{m}-x_{n}\right\| \leq \sum_{i=n}^{m-1}\left\|x_{i+1}-x_{i}\right\| \leq \sum_{i=n}^{m-1} \theta_{0}^{i-n_{0}}\left\|x_{n_{0}+1}-x_{n_{0}}\right\| .
$$

Since $\theta_{0}<1$, it follows from (3.20) that $\left\|x_{m}-x_{n}\right\| \rightarrow 0$ as $n \rightarrow \infty$ and hence $\left\{x_{n}\right\}$ is a Cauchy sequence in $X$. Let $x_{n} \rightarrow x^{*}$. It follows from (3.14) that $\left\{u_{n}\right\}$ is also a Cauchy sequence in $X$ and so we can suppose that $u_{n} \rightarrow u^{*} \in E$. Now we show that $u^{*} \in T\left(x^{*}\right)$. In fact, noting that $u_{n} \in T\left(x_{n}\right)$, we have

$$
\begin{aligned}
d\left(u^{*}, T x^{*}\right) & =\inf \left\{\left\|u_{n}-y\right\|: y \in T\left(x^{*}\right)\right\} \leq\left\|u^{*}-u_{n}\right\|+d\left(u_{n}, T\left(x_{n}\right)\right) \\
& \leq\left\|u^{*}-u_{n}\right\|+\hat{\mathbf{H}}\left(T\left(x_{n}\right), T\left(x^{*}\right)\right) \\
& \leq\left\|u^{*}-u_{n}\right\|+\gamma\left\|x_{n}-x_{n-1}\right\| \longrightarrow 0 .
\end{aligned}
$$

Hence $d\left(u^{*}, T\left(x^{*}\right)\right)=0$ and so $u^{*} \in T\left(x^{*}\right)$.

By continuity, $x^{*}, u^{*}$ satisfy

$$
g\left(x^{*}\right)=R_{\eta, M}^{\rho \lambda, A}\left[A\left(g\left(x^{*}\right)\right)-\rho\left(f\left(x^{*}\right)+u^{*}\right)\right] .
$$

By Lemma 3.1, now we know that $\left(x^{*}, u^{*}\right)$ is a solution of problem (2.25). This completes the proof.

From Theorem 3.4, we have the following results.

Theorem 3.5. Let $X$ be a q-uniformly smooth Banach space and let $A: X \rightarrow X$ be $r$ strongly $\eta$-accretive and $\epsilon$-Lipschitz continuous, respectively. Suppose that $T: X \rightarrow \mathrm{CB}(X)$ is $\gamma$ - $\hat{\mathbf{H}}$-Lipschitz continuous, $\eta: X \times X \rightarrow X$ is $\tau$-Lipschitz continuous, $M: X \rightarrow 2^{X}$ is $(A, \eta)$ accretive, and $f$ is $(e, \delta)$-relaxed cocoercive with respect to $A$ and $\sigma$-Lipschitz continuous. If

$$
\begin{gathered}
\rho<\frac{r}{m+\gamma \tau^{q-1}}, \\
\epsilon^{q}-q \rho \delta+q \rho e \sigma^{q}+c_{q} \rho^{q} \sigma^{q}<\left((r-\rho m) \tau^{1-q}-\rho \gamma\right)^{q},
\end{gathered}
$$

where $c_{q}$ is the constant as in Lemma 2.1, then the iterative sequences $\left\{x_{n}\right\}$ and $\left\{u_{n}\right\}$ generated by Algorithm 3.3 converge strongly to $x^{*}$ and $u^{*}$, respectively, and $\left(x^{*}, u^{*}\right)$ is a solution of problem (2.25).

Remark 3.6. (1) In problem (2.25), if $\mathrm{M}$ is an $(H, \eta)$-accretive operator or other the existing accretive operator in Banach space, $g$ is strongly accretive, and $f$ is $\delta$-strongly accretive with respect to $g_{1}$, then we can obtain the corresponding results of Theorems 3.4 and 3.5 (see, e.g., [2, Theorems 5.1 and 6.1] and the results of $[5,6,8]$, and the references therein).

(2) In problem (2.25), if $M$ is an $A$-monotone operator or other the existing monotone operator in Hilbert space, $g$ is strongly monotone, and $f$ is $\delta$-strongly monotone with respect to $g_{1}$, then we can obtain the corresponding results of Theorems 3.4 and 3.5 (see, e.g., [15, Theorem 1], [16, Theorem 1], and [12, Theorems 3.1 and 4.1]).

Thus, our results improve and generalize the corresponding results of recent works. 


\section{Acknowledgments}

The author acknowledges the support of the Educational Science Foundation of Sichuan Province (2004C018). The author is thankful to the referees for valuable suggestions.

\section{References}

[1] X. P. Ding, Existence and algorithm of solutions for generalized mixed implicit quasi-variational inequalities, Applied Mathematics and Computation 113 (2000), no. 1, 67-80.

[2] Y.-P. Fang, Y. J. Cho, and J. K. Kim, $(H, \eta)$-accretive operators and approximating solutions for systems of variational inclusions in Banach spaces, to appear in Nonlinear Analysis.

[3] Y.-P. Fang and N.-J. Huang, H-monotone operator and resolvent operator technique for variational inclusions, Applied Mathematics and Computation 145 (2003), no. 2-3, 795-803.

[4] __ Approximate solutions for nonlinear operator inclusions with $(H, \eta)$-monotone operators, Research Report, Sichuan University, Chengdu, 2003.

[5] _ $\quad H$-accretive operators and resolvent operator technique for solving variational inclusions in Banach spaces, Applied Mathematics Letters 17 (2004), no. 6, 647-653.

[6] __ Iterative algorithm for a system of variational inclusions involving $H$-accretive operators in Banach spaces, Acta Mathematica Hungarica 108 (2005), no. 3, 183-195.

[7] Y.-P. Fang, N.-J. Huang, and H. B. Thompson, A new system of variational inclusions with $(H, \eta)$ monotone operators in Hilbert spaces, Computers \& Mathematics with Applications 49 (2005), no. 2-3, 365-374.

[8] N.-J. Huang, Nonlinear implicit quasi-variational inclusions involving generalized $m$-accretive mappings, Archives of Inequalities and Applications 2 (2004), no. 4, 413-425.

[9] N.-J. Huang and Y.-P. Fang, Generalized m-accretive mappings in Banach spaces, Journal of Sichuan University 38 (2001), no. 4, 591-592.

[10] _ A new class of general variational inclusions involving maximal $\eta$-monotone mappings, Publicationes Mathematicae Debrecen 62 (2003), no. 1-2, 83-98.

[11] H.-Y. Lan, Y. J. Cho, and R. U. Verma, On nonlinear relaxed cocoercive variational inclusions involving $(A, \eta)$-accretive mappings in Banach spaces, to appear in Computers \& Mathematics with Applications.

[12] H.-Y. Lan, N.-J. Huang, and Y. J. Cho, A new method for nonlinear variational inequalities with multi-valued mappings, Archives of Inequalities and Applications 2 (2004), no. 1, 73-84.

[13] S. B. Nadler Jr., Multi-valued contraction mappings, Pacific Journal of Mathematics 30 (1969), 475-488.

[14] R. U. Verma, A-monotonicity and applications to nonlinear variational inclusion problems, Journal of Applied Mathematics and Stochastic Analysis 2004 (2004), no. 2, 193-195.

[15] _ Approximation-solvability of a class of A-monotone variational inclusion problems, Journal of the Korean Society for Industrial and Applied Mathematics 8 (2004), no. 1, 55-66.

[16] H. K. Xu, Inequalities in Banach spaces with applications, Nonlinear Analysis 16 (1991), no. 12, $1127-1138$.

[17] E. Zeidler, Nonlinear Functional Analysis and Its Applications II: Monotone Operators, Springer, Berlin, 1985.

Heng-You Lan: Department of Mathematics, Sichuan University of Science \& Engineering, Zigong, Sichuan 643000, China

E-mail addresses: hengyoulan@163.com; lanhengyou@suse.edu.cn 